\title{
Biomechanics analysis of human walking with load carriage
}

\author{
Xiuxia Yang*, Guorong Zhao, Di Liu, Weiei Zhou and Hewei Zhao \\ Department of Control Engineering, Naval Aeronautical and Astronautical University, Yantai, \\ Shandong, China
}

\begin{abstract}
.
BACKGROUND: Comprehensive analysis of the inherent laws and the biomechanic principles of human walking with load carriage and building kinematics, and kinematics model of human walking with load carriage, are very meaningful for the development of devices and apparatus that are related to human walking with load carriage, such as a lower limb exoskeleton. OBJECTIVE: The gait experiment of human walking with load carriage is designed and performed in this paper.

METHODS: The obtained video is marked and analyzed by using SIMI motion analytical software. The space motion coordinates at each body's mark point that is needed in the kinematics model of established human walking with load carriage is obtained. Based on inverse kinematics, a dynamic model of human walking with load carriage is established. The SPSS statistical analysis software is used for statistical processing for determining key gait parameters.

RESULTS: The influence of load and speed on the walking gait parameters is analyzed systematically.

CONCLUSIONS: The method provides a theoretical basis for the design of an exoskeleton.
\end{abstract}

Keywords: Human walking with load carriage, load experiment, biomechanics model, gait parameter

\section{Introduction}

The kinematic analysis of the human gait while carrying a load is the basis of many devices and apparatus such as the lower extremity exoskeleton. The theoretical basis has provided for the research and design of exoskeleton suits through the comprehensive investigation of the laws and the biomechanic principles that govern human ambulation with load carriage and analyzing the kinematic characteristics [1]. The extent of the research that characterizes load carriage will directly determine humanoid robot design. Robot design is of utmost importance for guiding the design of mechanical and electronic products, sports rehabilitation equipment and so forth [2].

The process of the human gait is simplified into Cavagna's inverted pendulum model [3]. This model accounts for many walking characteristics, such as the arch form trajectory of the body's center of gravity and the transformation of kinetic energy and potential energy during the single support. However, the highest theoretical efficiency of energy conversion is only $60 \%$ [4], and changes with the change of the length and the rate of stride. Recently, the research based on the inverted pendulum model shows that mechanical energy is mainly consumed during the change of the body's center of gravity [5].

\footnotetext{
${ }^{*}$ Corresponding author: Xiuxia Yang, Department of Control Engineering, Naval Aeronautical and Astronautical University, Yantai, Shandong, China. Tel.: +86 0535 6635639; E-mail: yangxiuxia@126.com.
} 
Through film analysis, researchers have begun to combine the walking kinematic parameters with the reaction force data from test force platforms, where the walking process is analyzed using an inverse kinematic method. In the past, restricted by research methods and means, the gait was primarily assessed using two-dimensional dynamics [6]. The procedure is relatively simple. Only one camera is used to shoot the walking motion in the sagittal plane. This method utilizes several marking points to define the two-dimensional coordinate system of each joint center [7].

In recent years, several articles have been published presenting the human gait using a threedimensional inverse kinematic analysis [8]9]. Incorporation of the three-dimensional analysis method is more complicated. Each marker requires at least two points to demark the position and orientation in a three dimensional space, and each mark point requires at least two cameras shooting from different angles to establish a three-dimensional coordinate. After establishing the marker's spatial location, the point is then used to determine the joint's motion in relation to a fixed position.

In order to determine the relationship between muscle contraction during walking and the joint movement, the kinematic method is used to quantitatively analyze the contribution of the lower limb muscles for vertical support, forward movement, and the swinging of the leg in a normal gait [10-12].

Load carriage is a special ambulatory condition. Due to the increased mass of the load on the bearer's back, the gait of a human body is changed accordingly. However, the impact of the load on the gait and kinematics is relatively small [13-15].

At present, the research of the human stride is primarily centered on kinematic analysis of the gait, and there are few literature reports of three-dimensional load carriage kinematics. Although there are some investigations on the influence of the load on the physiology in reference [1], the research on the effects of external weight on the human stride is not very extensive. Therefore, in this paper, gait experiments under load carriage were designed, and the biomechanics that dictate these processes were deeply characterized. The kinematics and kinematic model of the load carriage process were established, and the influence of the load and speed on gait parameters were analyzed systematically. The necessary theoretical basis is provided for the design of exoskeletons.

\section{The dynamic model of human lower limb for walking load carriage}

Generally, there are two avenues for dynamic modeling of physiological exercise: the power method and the inverse power method.

For this study, the inverse kinematic method was used to model the walking process, as this approach is suitable for models of walking with and without load bearing. The kinematic coordinates, the human body inertial parameters, gait, and the ground reaction force measured by the test force platform, are the input. The Newton-Euler equation was used to calculate the kinematics and kinematic parameters of each joint of the lower limbs. The flow chart for this process is shown in Fig. 1. The isolation method is used in conjunction with Newton-Euler's equation to establish the dynamic equation of each step. The corresponding equation of each joint was set up in the order in turn up from the feet.

Because the measured parameters are known quantities, the dynamic data of each joint are already solved. For example, Newton's second law was used for the foot. The kinematic equation of foot was established, and the force and torque of the foot exerted by the leg were calculated. According to Newton's third law, the magnitude of the force and torque of foot exerted by the leg are equal to the force and torque of the leg that is exerted by foot, however they are opposite in direction. Thus, the kinematic equations of the leg, knee and hip joints were also calculated by repeating this process. 


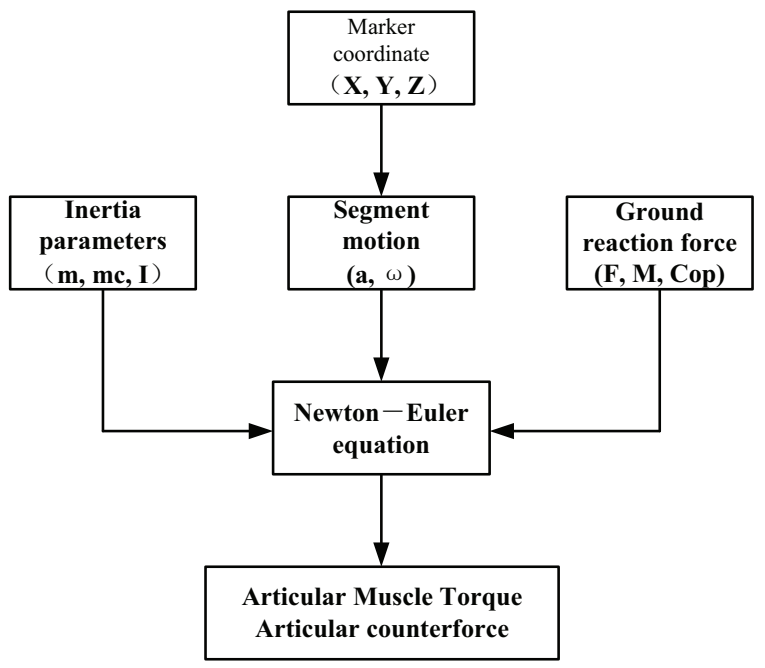

Fig. 1. Flow chart of the modeling process using inverse kinematics.

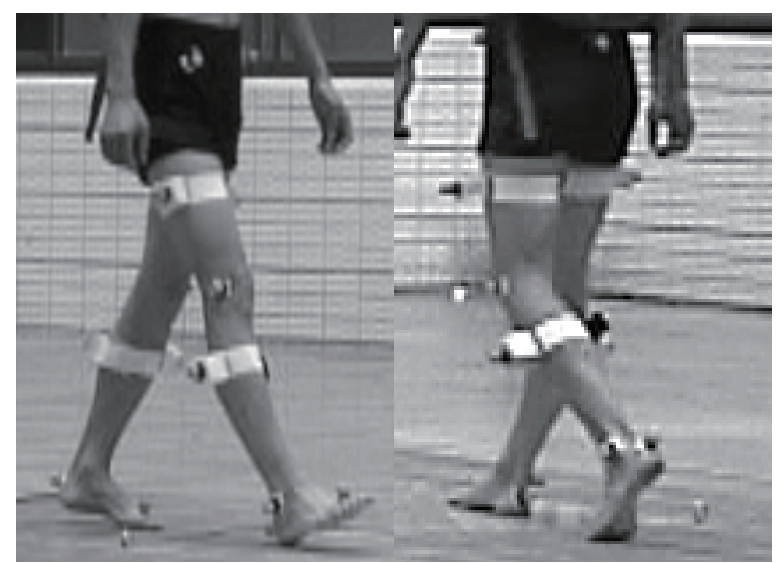

Fig. 2. The attachment of the markers.

\subsection{The calculation of joint's center}

This paper adopts the method that was proposed by Vaughan [6] where fifteen demarcation points were used to track the motion of each joint. $7-10 \mathrm{~cm}$ rod logos were bound on the tuberosity of the thigh and calf, and the mark points were placed on the top. This design ensures accurate motion tracking of each joint within a three-dimensional space, and is easily recorded.

The locations $p_{\text {Hip }}, p_{\text {Knee }}, p_{\text {Ankle }}, p_{\text {Toe }}$ of the hip joint, knee joint, ankle and toe can be calculated based on the location measurement of the points, according to reference [4].

\subsection{Angular motion of the joints}

\subsubsection{Defining the joint angle}

The joint angle is defined as the rotating angle of the distal joint in relation to the proximal joint. The specific definition is as follows.

Flexion/Stretching shaft $k$ is defined as the horizontal axis of the proximal joint. Flexion/Stretching angle is expressed by $\alpha$. Flexion (plantar flexion) is positive. Stretching (dorsiflexion) is negative.

The intorsion/extorsion axis $i$ is defined as the vertical axis of the distal joint. The intorsion/extorsion angle is expressed by $\beta$. The intorsion is positive and extorsion is negative.

The extented/adduction axis $l$ is defined as the axis which is vertical with the $k$ axis and the $i$ axis. The extented/adduction angle is expressed by $\gamma$. The extented is positive and adduction is negative.

\subsubsection{The motion of the Euler angle}

The right leg is taken as an example to specify the definition of the Euler angle. First the right calf's center of gravity is translated with respect to a fixed terrestrial origin. Then, three rotations are performed in turn.

1. Rotating the angle $\phi$ around the $X$ axis;

2. Rotating the angle $\theta$ around the $Y$ axis;

3. Rotating the angle $\psi$ around the $Z$ axis. 
The three rotation angles are designated as the Euler angles. The angular velocity of the joint's motion can be calculated by the change in the Euler angle. Then, the angular acceleration of joint's motion can be obtained by taking the derivative of angular velocity.

The measured parameters are regarded as known variables to calculate the kinematic data. Using Newton's second law, the kinematic equation of foot is established. The force and torque the shank exerts on the foot can be calculated. From Newton's third law, it is known that the force and torque that the shank exerts on the foot are equal to the force and the torque that the foot exerts on the shank, but opposite in direction. Thus, the force and torque of knee joint can be calculated by establishing the kinematic equations of the shank. Similarly, the force and torque of the hip joint can be calculated by establishing the kinematic equations of the thigh.

\section{The gait experiment of human walking with load carriage}

The load bearing gait was modeled by using the inverse power method. There are three kinds of known parameters: the human body kinematic parameters, the kinematic coordinates of the mark point, and the measurement data of the test force platform. These parameters can be obtained through physical measurement and gait experiments.

\subsection{The measurement and calculation of human body parameters}

To accommodate the research needs in this paper, fifteen basic measuring parameters were selected. In order to improve the accuracy of the image resolution, the experimental subjects were shirtless and wore dark tight shorts. Fifteen reflective markers balls with a $2 \mathrm{~cm}$ diameter were attached to every segment of the lower limbs and pelvis. The specific configuration is shown in Fig. 2.

\subsection{The gait experiment}

\subsubsection{The acquisition of motion parameters}

In the process of load carriage, two kinds of information should be recorded. One is kinematic information, that is, the position coordinates of each marker in space. The other is dynamic information, which is the acting force between the human body and the ground. Video analysis systems obtain kinematic data through analysis of the captured image. For the dynamic information, a multi-component force platform is used to obtain the components of force that the body imparts on the ground. Then, the motion variables of the subjects can be calculated using the motion analysis software.

\subsubsection{Experimental scheme and process}

(1) The subjects

Ten men, age $24.5 \pm 1.5$ were chosen as test subjects. They were in good health and had heights $171.2 \pm 4.2 \mathrm{~cm}$ and masses $64.1 \pm 15.9 \mathrm{~kg}$.

(2) The experimental scheme and process

The experimental scheme is shown in Fig. 3. Experiment employed four shot frequencies: $84 \mathrm{~Hz}$, $96 \mathrm{~Hz}, 108 \mathrm{~Hz}$ and $120 \mathrm{~Hz}$. The control step, that is, the distance of the subject's step, was approximately $70 \mathrm{~cm}$. The speed of subjects was about $0.98 \mathrm{~m} / \mathrm{s}, 1.12 \mathrm{~m} / \mathrm{s}, 1.26 \mathrm{~m} / \mathrm{s}$ and $1.4 \mathrm{~m} / \mathrm{s}$, respectively, the load was set as $10 \mathrm{~kg}, 20 \mathrm{~kg}$, and $30 \mathrm{~kg}$. 


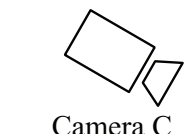

Camera C
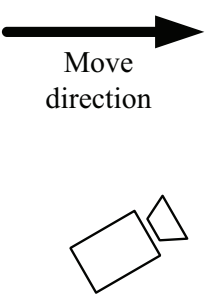

Camera D

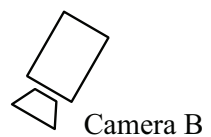

Force

board

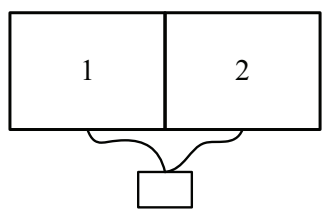

Force platform

monitors computer
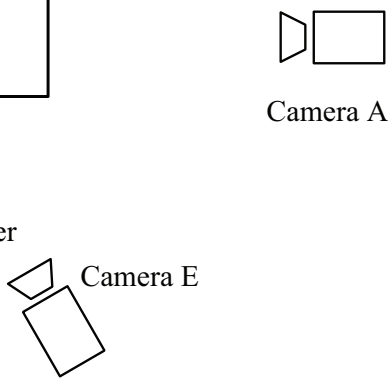

Camera A

Fig. 3. Scheme of the gait experiment.

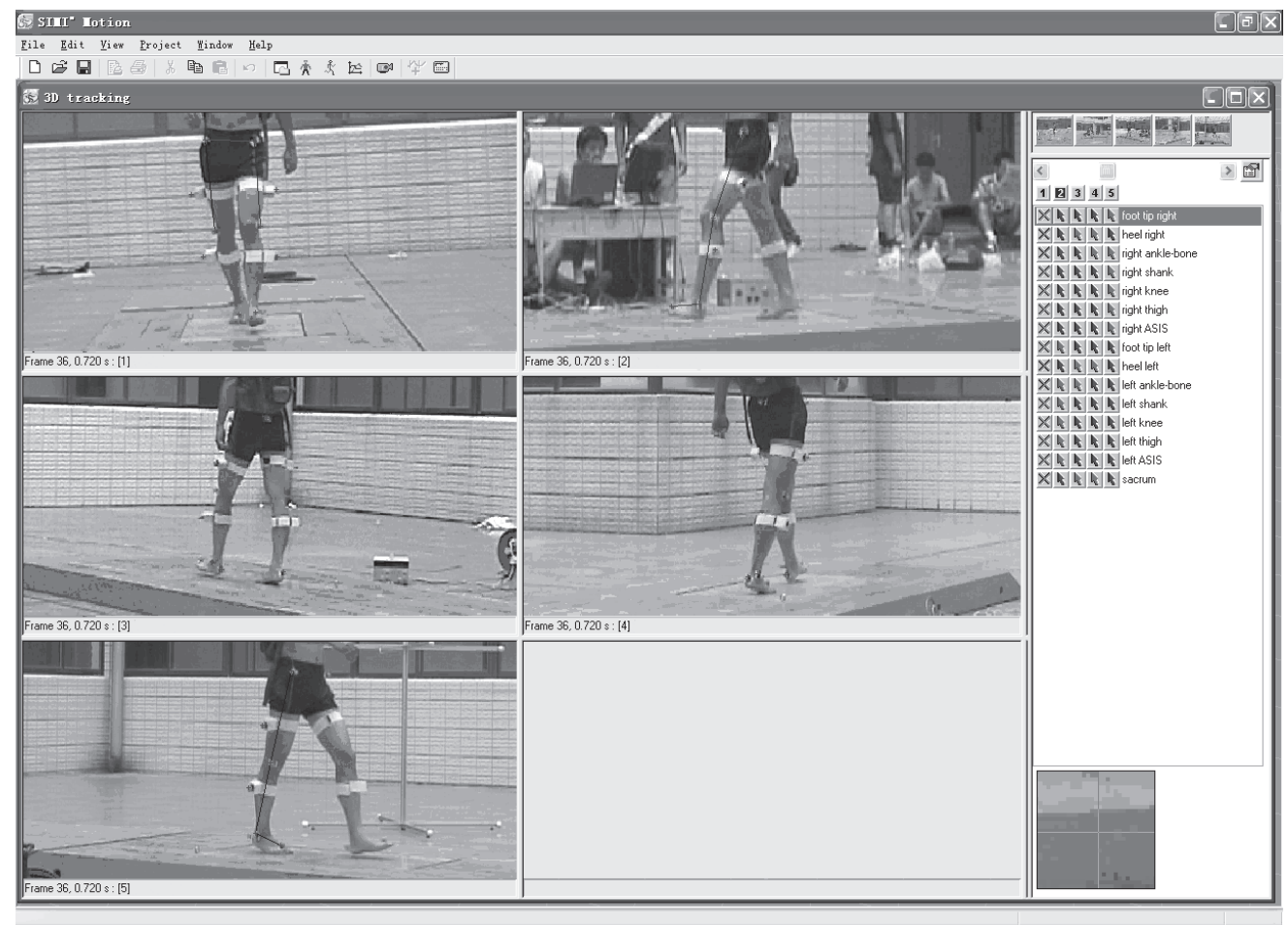

Fig. 4. Marker analysis.

\subsubsection{Video resolution}

The footage was analyzed using SIMI movement analysis software. The image information taken from the mark point can be converted into spatial coordinate data by analysis software calculation. This is illustrated in Fig. 4. 


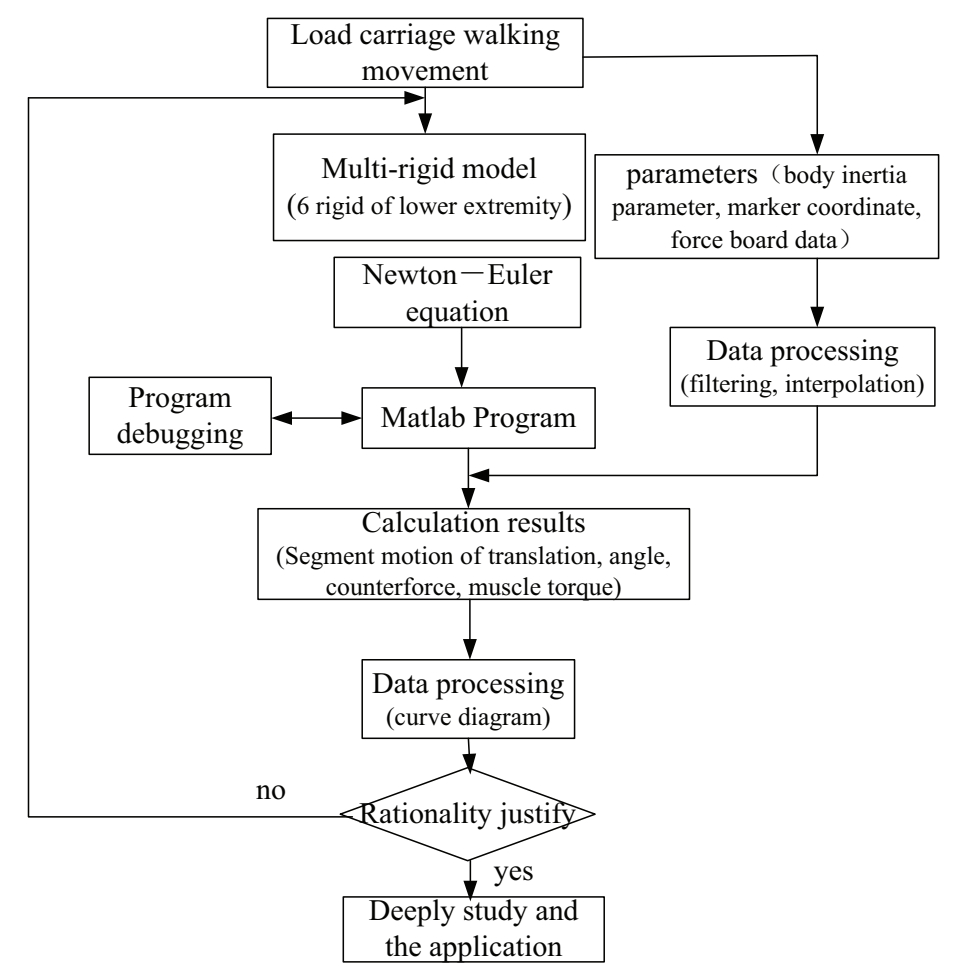

Fig. 5. Flow chart of the simulation process.

\subsection{The processing of experimental data}

\subsubsection{Data smoothing}

The raw coordinate data was generated from the video analysis. Processing of the original data must be undertaken due to the presence of noise. Using a second-order digital filter, as established by Butterworth, a cutoff frequency of $5 \mathrm{~Hz}$ to $8 \mathrm{~Hz}$ is generally employed for denoising walking data. A $6 \mathrm{~Hz}$ cutoff frequency was selected for this study. However, applying a filter can generate phase discrepancies, and to eliminate these phase differences, a correcting value was filtered in the opposite direction.

\subsubsection{Data interpolation}

A $50 \mathrm{f} / \mathrm{s}$ frame rate was used to record the process. The interpolation was done after the original data filtration and smoothing, and the sampling frequency was increased to $200 \mathrm{~Hz}$ which improved the accuracy of the differential.

\section{The kinematic simulation of load carriage}

A variety of load carriage kinematics and kinematic parameters can be calculated by from basic human body parameters and mark point kinematic coordinates. The flow chart of the simulation process is shown in Fig. 5.

The effects of different loads and speeds on gait parameters are listed below. Time is expressed as a percentage of the gait cycle. 


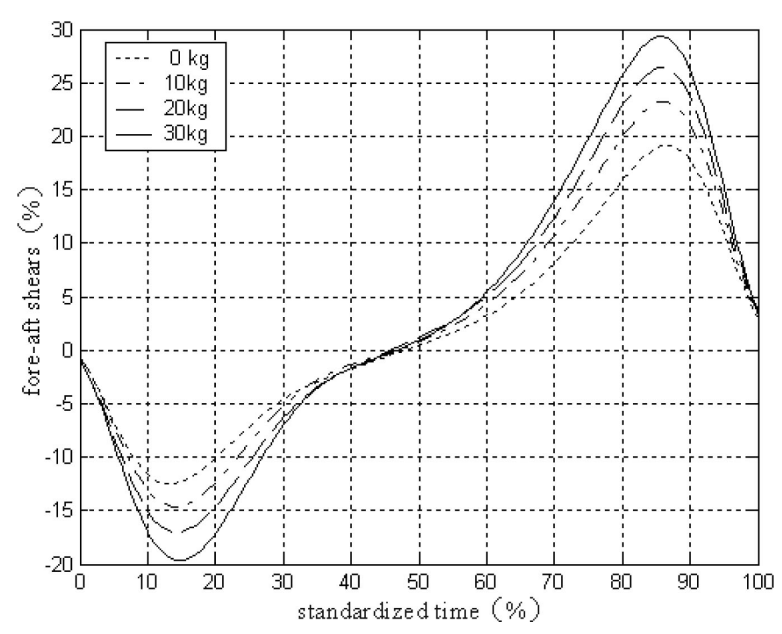

Fig. 6. Fore-aft shears under different loads.

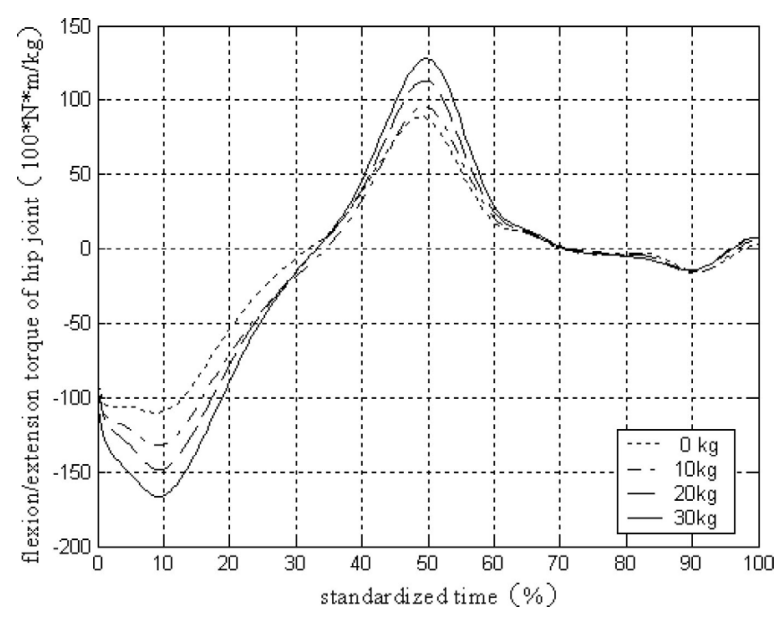

Fig. 8. Hip flexion and extension torques under different loads.

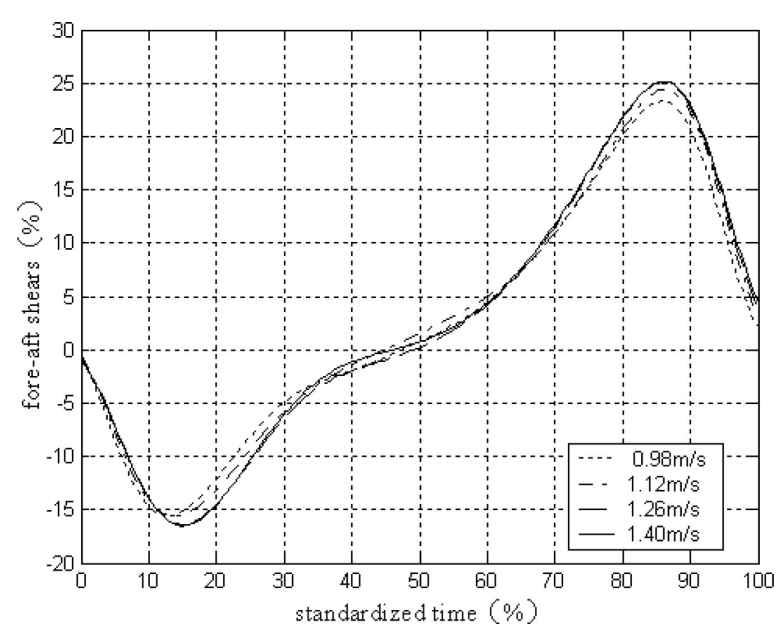

Fig. 7. Fore-aft shears at different speeds.

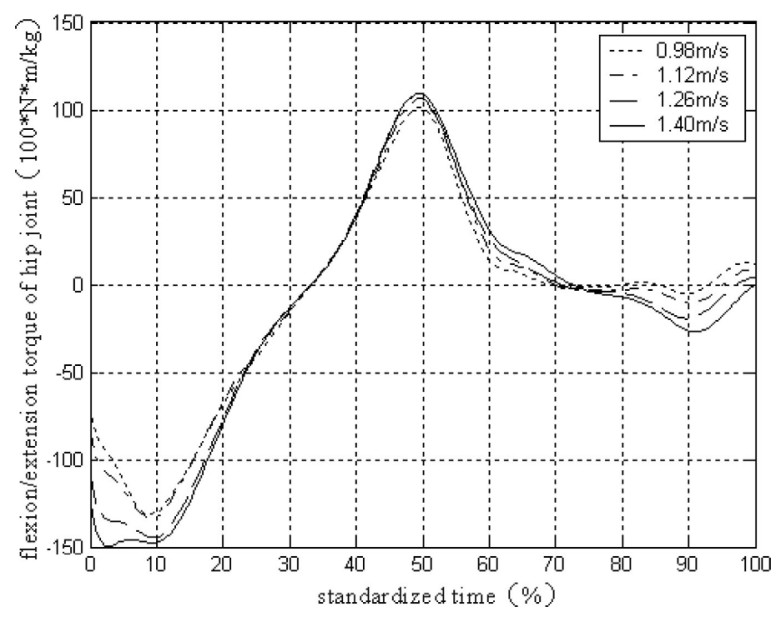

Fig. 9. Hip flexion and extension torques at different speeds.

\subsection{The anterior-posterior ground reaction force component}

Behavior of the fore-aft shears under different conditions are shown in Figs 6 and 7.

As evidenced in Fig. 6, the amplitude of the fore-aft shears increases significantly with the increase of the load. A certain linear relation emerges in Fig. 6. The load was increased in $10 \mathrm{~kg}$ increments, and the growth of the amplitude of the aft shear was about $2 \%$ of the weight; the amplitude growth of the fore shear was about $4 \%$ of the weight. There was a strong proportionality between the increase of the load and the amplitude growth of the fore-aft shears. It should be noted that the fore shear's amplitude growth was greater than that of the aft shear. In Fig. 7, an increasing trend of the fore-aft shears was not observed with an increase of speed.

\subsection{Joint muscle torque}

The curves of the hip flexion torque are shown in Figs 8 and 9. 


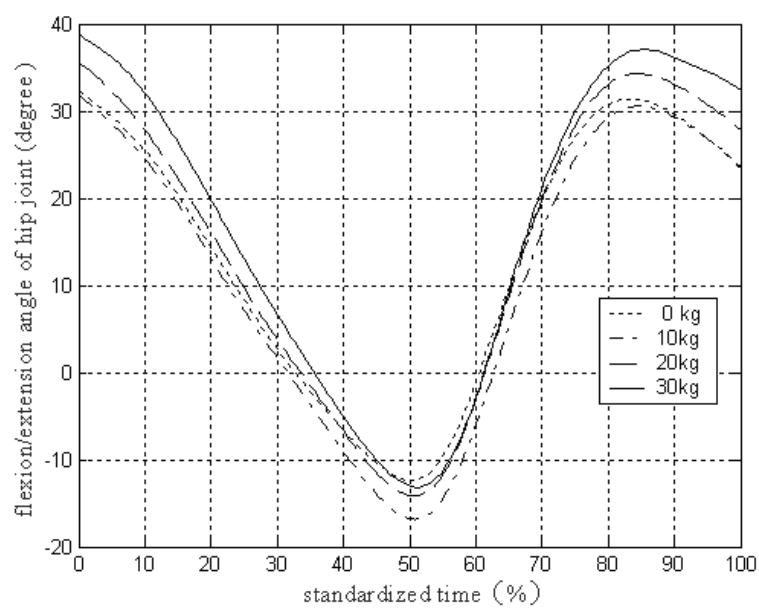

Fig. 10. The hip joint angle under different loads.

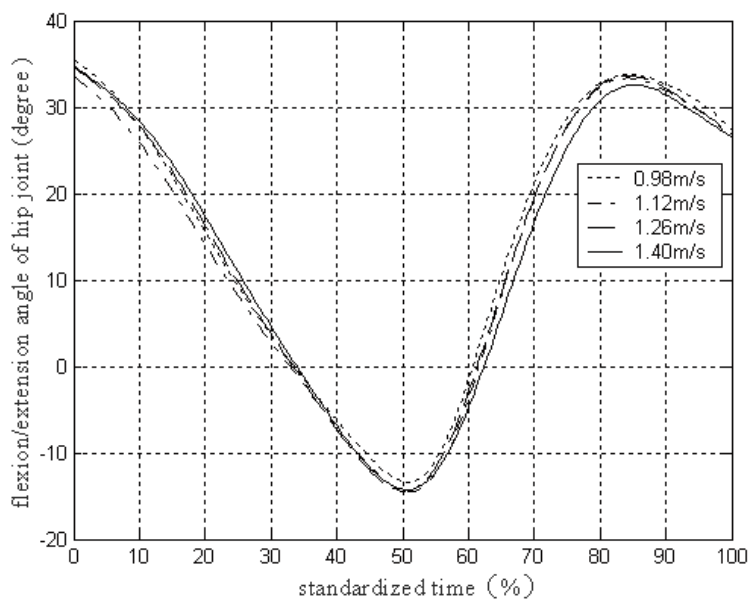

Fig. 11. The hip joint angle at different speeds.

It can be seen in Fig. 8 that the load mass exerts great influence on the flexion and extension torque of the hip joint. In the swing phase, the flexion and extension torques are not affected by load variation. The stretch torque of the hip joint increases to support greater body weight with the increase of load.

In Fig. 9 it can be seen that the impact of the change of speed on the flexion and extension torque of the hip joint is mainly reflected in the early stages of the stance phase and the early and late portion of the swing phase. The influence on other stages of the gait cycle is small.

\subsection{The angle of the hip joint}

The flexion angles of the hip joint under different conditions are shown in Figs 10 and 11.

It can be seen in Fig. 10 that the flexion angle of the hip joint produces an apparent change when a $10 \mathrm{~kg}$ load was added. With additional mass, the bending angle increased gradually over the course of the gait cycle, and was notable when the heel touched down. While the bearing external weight, the body maintained its center of gravity over the pelvis to reduce the energy consumption. The front rake of the trunk increased, which lead to the increase of the bending angle. When the heel touched the ground, the increase of the flexion angle enlarged the buffer between the heel and ground. With every $10 \mathrm{~kg}$ incremental increase, the bending angle increased by $3.5^{\circ}$.

In Fig. 11, it is observed that the influence of walking speed on the flexion angle of the hip joint was less significant compared to the load mass.

\section{Conclusion}

The biomechanical model of human walking with load carriage was analyzed kinematically. Experiment data of load carriage was used the basis for this investigation, and the dynamic data of each lower limb segment was calculated. This paper establishes a detailed theoretical basis for engaging in future research the human gait under load carriage. 


\section{Acknowledgement}

This research is supported by the General Armament Department Pre-research Foundation of China under Grant No9140A26020313JB14370.

\section{References}

[1] Adam B. Zoss H. Kazerooni AC. Biomechanical design of the berkeley lower extremity exoskeleton (BLEEX). IEEE/ASME Transactions on Mechatronics 2006; 11(2): 128.

[2] Kim WS, Lee SH, Lee HD, Yu SN, Han JS, Han CS. Development of the heavy load transferring task oriented exoskeleton adapted by lower extremity using qausi-active joints. ICROS-SICE International Joint Conference, Fukuoka, Japan, 2009.

[3] Cavagna GA, Margaria R. Mechanics of walking. Journal of Applied Physiology 1966; 21: 271.

[4] Minetti AE, Capelli C, Zamparo P. Effects of stride frequency on mechanical power and energy expenditure of walking. Medicine and Science in Sports and Exercise 1995; 27: 1194.

[5] Zheng X. The human body measurement and evaluation. Beijing: Higher education press; 1990. p. 107.

[6] Donelan JM, Kram R, Kuo AD. Mechanical work for step-to-step transitions is a major determinant of the metabolic cost of human walking. Journal of Experimental Biology 2002; 205: 3717.

[7] Kong K, Masayoshi T. A gait monitoring system based on air pressure sensors embedded in a shoe. IEEE/ASME Transactions on Mechatronics 2009; 14(3): 358.

[8] Tine A, Erik BS, Poul DP. Comparison of inverse kinematics calculated by two- and three-dimensional models during walking. Gait and Posture 2001; 13: 73.

[9] Allard P, Lachance R, Aisaoui R, Duhaime M. Simultaneous bilateral 3-D able-bodied gait. Human Movement Science 1996; 15: 327.

[10] Eng JJ, Winter DA. Kinetic analysis of the lower limbs during walking: what information can be gained from a threedimensional model. Journal of Biomechanics 1995; 28: 753.

[11] Neptune RR, Kautz SA, Zajac FE. Contributions of the individual ankle plantar flexors to support forward progression and swing initiation during walking. Journal of Biomechanics 2001; 34: 1387.

[12] Anderson FC, Pandy MG. Individual muscle contributions to support in normal walking. Gait \& Posture 2003; $17: 15$.

[13] Anderson FC, Goldberg SR, Pandy MG, et al. Contributions of Muscle Forces and Toe-off Kinematics to Peak Knee Flexion during the Swing Phase of Normal Gait: an Induced Position Analysis. Journal of Biomechanics 2004; $37: 731$.

[14] Knapik J, Harman E, Reynolds K. Load carriage using packs: A review of physiological biomechanical and medical aspects. Applied Ergonomics 1996; $27: 207$.

[15] Harman E, Han K-H, Frykman P, et al. The Effects of Backpack Weight on the Biomechanics of Load Carriage. Technical Report No. T00-17, MA: US Army Research Institute of Environmental Medicine. 2000. 